\title{
Right circulant matrices with Jacobsthal sequence
}

\author{
Aldous Cesar F. Bueno \\ Department of Mathematics and Physics, Central Luzon State University \\ Science City of Muñoz 3120, Nueva Ecija, Philippines \\ E-mail: aldouz_cezar@yahoo.com
}

\begin{abstract}
In this paper, the eigenvalues, Euclidean norm and inverse of right circulant matrices with Jacobsthal sequence were obtained.
\end{abstract}

Keywords: Jacobsthal sequence, right circulant matrix

\section{Introduction}

The Jacobsthal sequence $\left\{j_{k}\right\}_{k=0}^{+\infty}$ satisfies the recurrence relation

$$
j_{k}=j_{k-1}+2 j_{k-2}
$$

with initial values $j_{0}=0$ and $j_{1}=1$.

The right circulant matrix as defined in [1] is given by

$$
\operatorname{RCIRC} C_{n}(\vec{j})=\left(\begin{array}{cccccc}
j_{0} & j_{1} & j_{2} & \cdots & j_{n-2} & j_{n-1} \\
j_{n-1} & j_{0} & j_{1} & \cdots & j_{n-3} & j_{n-2} \\
j_{n-2} & j_{n-1} & j_{0} & \cdots & j_{n-4} & j_{n-3} \\
\vdots & \vdots & \vdots & \ddots & \vdots & \vdots \\
j_{2} & j_{3} & j_{4} & \cdots & j_{0} & j_{1} \\
j_{1} & j_{2} & j_{3} & \cdots & j_{n-1} & j_{0}
\end{array}\right)
$$

where $j_{k}$ are the first $\mathrm{n}$ terms of the Jacobsthal sequence.

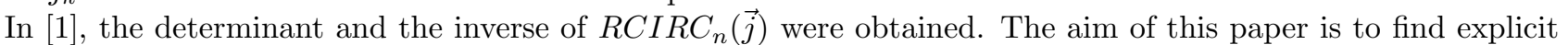
forms for the eigenvalues, Euclidean norm and inverse of $R C I R C_{n}(\vec{j})$. In the inverse, we will be using Inverse Discrete Fourier Transform.

\section{Preliminary notes}

Lemma 2.1
where $\omega=e^{2 \pi i / n}$

Proof:

$$
\sum_{k=0}^{n-1}\left[\frac{2^{k}-(-1)^{k}}{3}\right] \omega^{-m k}=\frac{1}{3}\left[\sum_{k=0}^{n-1}\left(2 \omega^{-m}\right)^{k}-\sum_{k=0}^{n-1}\left(-\omega^{-m}\right)^{k}\right]
$$




$$
\begin{aligned}
& =\frac{1}{3}\left[\frac{1-\left(2 \omega^{-m}\right)^{n}}{1-2 \omega^{-m}}-\frac{1-\left(-\omega^{-m}\right)^{n}}{1-\left(\omega^{-m}\right)}\right] \\
& =\frac{1}{3}\left[\frac{1-2^{n}}{1-2 \omega^{-m}}-\frac{1-(-1)^{n}}{1+\omega^{-m}}\right]
\end{aligned}
$$

Lemma 2.2 Given the equation

$$
s_{k}=\sum_{k=0}^{n-1}\left[r \omega^{-m}-1\right] \omega^{m k}
$$

where $r$ is non-zero,

$$
\begin{aligned}
& s_{0}=-n \\
& s_{1}=r n \\
& s_{k}=0 ; \text { for } k \geq 2
\end{aligned}
$$

Proof:

For $\mathrm{k}=0$

$$
\begin{aligned}
s_{0} & =\sum_{k=0}^{n-1}\left[r \omega^{-m}-1\right] \\
& =\frac{r\left(1-\omega^{n}\right)}{1-\omega}-n \\
& =-n
\end{aligned}
$$

For $\mathrm{k}=1$

$$
\begin{aligned}
s_{1} & =\sum_{k=0}^{n-1}\left[r \omega^{-m}-1\right] \omega^{m} \\
& =r n-\frac{1-\omega^{n}}{1-\omega} \\
& =r n
\end{aligned}
$$

For $k \geq 2$

$$
\begin{aligned}
s_{k} & =\sum_{k=0}^{n-1}\left[r \omega^{-m}-1\right] \omega^{m k} \\
& =\sum_{k=0}^{n-1}\left[r \omega^{m(k-1)}-\omega^{m k}\right] \\
& =r \frac{1-\omega^{n(k-1)}}{1-\omega^{k-1}}-\frac{1-\omega^{n}}{1-\omega} \\
& =0
\end{aligned}
$$

\section{Main results}

Theorem 3.1 If $n$ is even, the eigenvalues of $R C I R C_{n}(\vec{j})$ are given by

$$
\lambda_{m}=\frac{1-2^{n}}{3-6 \omega^{-m}}
$$

where $m=0,1 \ldots, n-1$. 
Proof:

The eigenvalues of a right circulant matrix are the Discrte Fourier Transform of the entries in the first row, hence

$$
\lambda_{m}=\sum_{k=0}^{n-1}\left[\frac{2^{k}-(-1)^{k}}{3}\right] \omega^{-m k}
$$

where $\mathrm{m}=0,1 \ldots, \mathrm{n}-1$. Using $(2)$ and $\mathrm{n}$ being even, we have

$$
\lambda_{m}=\frac{1-2^{n}}{3-6 \omega^{-m}}
$$

Theorem 3.2 If $n$ is odd, the eigenvalues of $R C I R C_{n}(\vec{j})$ are given by

$$
\lambda_{m}=\frac{1-2^{n}}{3-6 \omega^{-m}}-\frac{2}{3+3 \omega^{-m}}
$$

where $m=0,1 \ldots, n-1$.

Proof:

The same process as the previous theorem but with $\mathrm{n}$ odd.

Theorem 3.3 The Euclidean norm of $R C I R C_{n}(\vec{j})$ is given by

$$
\left\|R C I R C_{n}(\vec{j})\right\|_{E}=\frac{\sqrt{n^{2}+3 n j_{n}^{2}}}{3}
$$

Proof:

$$
\begin{aligned}
\left\|R C I R C_{n}(\vec{j})\right\|_{E} & =\sqrt{\sum_{i=1, j=1}^{n} a_{i j}^{2}} \\
& =\sqrt{n \sum_{k=0}^{n-1} j_{k}^{2}} \\
& =\frac{1}{3} \sqrt{n \sum_{k=0}^{n-1}\left[4^{k}+(-2)^{k+1}+1\right]} \\
& \left.=\frac{1}{3} \sqrt{n\left[\frac{4^{n}-1}{3}+\frac{2+(-2)^{n+1}}{3}+n\right.}\right] \\
& =\frac{1}{3} \sqrt{n^{2}+n\left[\frac{4^{n}+(-2)^{n+1}+1}{3}\right]} \\
& =\frac{1}{3} \sqrt{n^{2}+3 n\left[\frac{2^{2 n}-2(-2)^{n}+1^{2 n}}{9}\right]} \\
& =\frac{1}{3} \sqrt{n^{2}+3 n\left[\frac{2^{n}-(-1)^{n}}{3}\right]^{2}} \\
& =\frac{\sqrt{n^{2}+3 n j_{n}^{2}}}{3}
\end{aligned}
$$

Theorem 3.4 If $n$ is even, $R C I R C_{n}^{-1}(\vec{j})$ is given by $R C I R C_{n}\left(s_{0}, s_{1}, \ldots, s_{n-1}\right)$ where

$$
\begin{aligned}
& s_{0}=\frac{3}{1-2^{n}} \\
& s_{1}=-\frac{6}{1-2^{n}} \\
& s_{k}=0 ; \text { for } k \geq 2 .
\end{aligned}
$$


Proof:

The inverse of a right circulant matrix is the Inverse Discrete Fourier Transform of the inverse of its eigenvalues, hence

$$
\begin{aligned}
s_{k} & =\frac{1}{n} \sum_{m=0}^{n-1} \lambda_{m}^{-1} \omega^{m k} \\
& =\frac{3}{n} \sum_{m=0}^{n-1}\left[\frac{1-2 \omega^{-m}}{1-2^{n}}\right] \omega^{m k} \\
& =-\frac{3}{\left(1-2^{n}\right) n} \sum_{m=0}^{n-1}\left[2 \omega^{-m}-1\right] \omega^{m k}
\end{aligned}
$$

Using Lemma 2.2, the thereom follows.

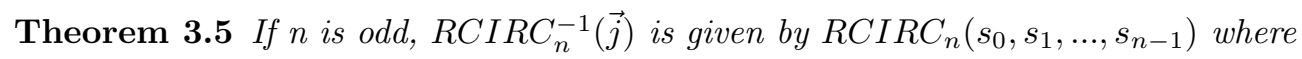

$$
s_{k}=\frac{3}{n} \sum_{k=0}^{n-1}\left[\frac{\left(5-2^{n}\right) \omega^{-m}-1}{\left(1-2 \omega^{-m}\right)\left(1+\omega^{-m}\right)}\right] \omega^{m k}
$$

Proof:

$$
\begin{aligned}
s_{k} & =\frac{1}{n} \sum_{m=0}^{n-1}\left[\frac{1-2^{n}}{3-6 \omega^{-m}}-\frac{2}{3+3 \omega^{-m}}\right]^{-1} \omega^{m k} \\
& =\frac{3}{n} \sum_{k=0}^{n-1}\left[\frac{1-2^{n}+\omega^{-m}-2^{n} \omega^{-m}-2+4 \omega^{-m}}{\left(1-2 \omega^{-m}\right)\left(1+\omega^{-m}\right)}\right] \omega^{m k} \\
& =\frac{3}{n} \sum_{k=0}^{n-1}\left[\frac{\left(5-2^{n}\right) \omega^{-m}-1}{\left(1-2 \omega^{-m}\right)\left(1+\omega^{-m}\right)}\right] \omega^{m k}
\end{aligned}
$$

\section{Conclusion}

The eigenvalues and the inverse of right circulant with Jacobsthal sequence take different forms depending on whether $\mathrm{n}$ is even or odd while the Euclidean norm doesn't. Furthermore, we have expressed the Euclidean norm in terms of $\mathrm{n}$ and $n^{\text {th }}$ Jacobsthal number.

\section{References}

[1] D. Bozkurt, "On the Determinants and Inverses of Circulant Matrices with Jacobsthal and Jacobsthal-Lucas Numbers", arXiv: 1202.1068v1 [math.NA] 29 Jan. 2012

[2] A.C.F. Bueno, "Right Circulant Matrices with Geometric Progression", International Journal of Applied Mathematical Research, Vol.1, No.4 (2012), 593-603

[3] H. Karner, J. Schneid, C. Ueberhuber, "Spectral decomposition of real circulant matrices", Linear Algebra and Its Applications, 367, (2003), 301-311 